\title{
PALESTRAS DE PREVENÇÃO DO CÂNCER NA COMUNIDADE DE SANTA MARIA
}

\author{
Caren Leivas Pozzer ${ }^{1}$ \\ Lissandra Dal Lago ${ }^{2}$ \\ Lucimara Volpato ${ }^{3}$
}

\section{RESUMO}

Projeto de extensão realizado pelos acadêmicos de Medicina da Universidade Federal de Santa Maria (RS) entre abril e junho de 2008, com o objetivo de promover a educação em prevenção do câncer à população da comunidade de Santa Maria, através de palestras sobre o tema, e verificar a efetividade das palestras. Antes das palestras, em quatro ocasiões, foram distribuídos questionários com questões objetivas sobre câncer e sua prevenção para os ouvintes, compostos pelos pacientes diabéticos e hipertensos das Unidades Básicas de Saúde de Santa Maria. Após as palestras, o mesmo instrumento foi distribuído para testar o conhecimento adquirido. Os dados foram analisados através de média, frequência e tabelas pelo programa Epi Info versão 6.04. A amostra final foi de 61 pessoas. Vinte e duas pessoas (36\%) tinham o Ensino Fundamental incompleto; oito (16\%) tinham Ensino Fundamental completo; 26 (43\%) tinham Ensino Médio completo e três (5\%) tinham Ensino Superior completo. Após a palestra, houve maior número de acertos nas questões sobre câncer de colo de útero, boca, estômago, mama, esôfago, próstata e bexiga. Nas questões sobre câncer de pele e intestino, houve um leve decréscimo no número de acertos e o conhecimento sobre câncer de pulmão não foi alterado após a palestra. As palestras se mostraram úteis à comunidade de Santa Maria, no entanto, precisamos adaptar continuamente a forma de exposição conforme o nível educacional dos expectadores, que, neste caso, era baixo.

Palavras-chave: Palestras. Prevenção. Câncer.

\footnotetext{
${ }^{1}$ Acadêmica do Curso de Medicina da Universidade Federal de Santa Maria, clpozzer@gmail.com

${ }^{2}$ Médica oncologista, doutora em Ciências Médicas, professora adjunta do Departamento de Clínica Médica do Centro de Ciências da Saúde da Universidade Federal de Santa Maria, dlissandra@hotmail.com

${ }^{3}$ Acadêmica do Curso de Medicina da Universidade Federal de Santa Maria luci,volpato@yahoo.com
} 


\title{
LECTURES ON THE PREVENTION OF CANCER IN THE COMMUNITY OF SANTA MARIA
}

\begin{abstract}
Outreach Project realized by the graduate students of Medicine from Federal University of Santa Maria, RS, Brazil, between April and June of 2008, with the objectives of promoting cancer prevention education among the community of Santa Maria, through lectures on the subject, and evaluating the efficacy of this educative approach. Four lectures were done about cancer prevention. Evaluation questionnaires were distributed to the target audience before and after the lectures. The data was summarized with frequency and average (tables from Epi Info version 6.04). From 61 persons, 22(36\%) and 8(16\%) had incomplete and complete primary school, respectively; 26 (43\%) had complete secondary school; and three (5\%) had completed superior education. After the lecture, there were more correct questions about cervical, head and neck, gastric, breast, esophageal, prostate and bladder cancer. There was a small decrease in the number of correct questions about skin and gastrointestinal cancer and no change in the number of correct answers about lung cancer. The lectures were useful for the community, however the way of displaying the subject has to be continued improving for the persons with low educational level.
\end{abstract}

Keywords: Lectures. Prevention. Cancer.

\section{INTRODUÇÃO}

Conforme a Organização Mundial de Saúde (OMS), as neoplasias estão entre as principais causas de morte em todo mundo e, em 2008, foi estimado que 12 milhões de pessoas tenham sido diagnosticadas por câncer. O câncer está em segundo lugar entre as principais causas de óbito em adultos, quando comparado com outras doenças crônicas nãotransmissíveis, como a doença cardiovascular e o diabetes mellitus (FERNANDES; BARACAT; LIMA, 2004). A estimativa da OMS é que no ano de 2020 o câncer será a principal causa de morte tanto nos países desenvolvidos quanto nos em desenvolvimento.

O câncer é uma doença passível de prevenção e quanto mais precoce sua detecção, maior a probabilidade de cura (DUNCAN, 2004). Há três tipos de abordagens preventivas no câncer: a primária, na qual a prevenção da doença acontece antes que ela se desenvolva; a 
secundária, na qual há a detecção e o tratamento precoce da doença na fase pré-clínica, impedindo a sua progressão; e a terciária, que consiste em atividades de reabilitação após a instalação da doença a fim de minimizar as complicações (GOLDMAN; AUSIELLO, 2005). A prevenção efetiva e o manejo do câncer nos serviços de atenção básica exigem o desenvolvimento de ações programáticas, que envolve em conjunto toda a equipe de saúde, além de entidades comunitárias fora do setor da saúde (DUNCAN, 2004).

As modalidades primárias para a prevenção do câncer exigem mudanças no comportamento, especialmente naquelas que envolvem fumo, álcool, dieta, atividade física e infecções relacionadas com a atividade sexual (GOLDMAN; AUSIELLO, 2005). Segundo a OMS, quarenta por cento dos cânceres podem ser prevenidos, principalmente pelo combate ao tabagismo, adoção de uma dieta saudável, pela prática de atividades físicas e pela prevenção de infecções de risco, como por exemplo, a infecção pelo papilomavírus humano (HPV) no câncer de colo uterino.

Segundo o Instituto Nacional do Câncer (INCA), o estabelecimento de medidas de controle ao câncer deve ser feito com base nos tipos de câncer mais incidentes da respectiva região. No Rio Grande do Sul (RS), nos homens, o câncer de próstata é o mais incidente, com uma perspectiva de 4.430 casos novos em 2008. Nas mulheres, o câncer de mama é o mais incidente com uma estimativa de 4.880 casos novos em 2008 e o de colo de útero está em segundo lugar, estimando-se 1.610 casos novos no mesmo ano. Os demais tipos mais incidentes no RS são: câncer de pulmão, câncer de cólon e reto, câncer de estômago, câncer de esôfago, câncer na cavidade oral e câncer de pele (INCA, 2008).

Existe uma boa evidência de que o estilo de vida tem impacto sobre o risco de câncer, e grandes mudanças de hábito de vida da população podem reduzir significativamente o número de casos da doença (CURRY, 2003 apud STEIN; COLDITZ, 2004). Por isso, este projeto teve como objetivo geral o de promover a educação em prevenção do câncer aos diabéticos e hipertensos atendidos pela Unidade Básica de Saúde Wilson Noal do bairro Camobi de Santa Maria e aos familiares e pacientes da Associação de Apoio às Pessoas com Câncer (AAPECAN) de Santa Maria. Além disso, verificou-se a efetividade das palestras sobre prevenção do câncer aplicadas para estes diferentes públicos.

\section{MATERIAL E MÉTODOS}


A população adstrita pela pesquisa foi composta pelos indivíduos que pertencem aos grupos de diabéticos e hipertensos da Unidade Básica de Saúde Wilson Noal, dos centros comunitários Santa Helena e Vila Jardim do bairro Camobi da cidade de Santa Maria, e pelos sócios da AAPECAN de Santa Maria, que estiveram presentes no encerramento da $1^{\text {a }}$ Semana de Prevenção Contra o Câncer, em outubro de 2008, evento promovido por esta entidade.

Entre abril de 2008 e junho de 2008, foram realizadas quatro palestras sobre prevenção ao câncer, uma na Unidade de Saúde Wilson Noal no bairro Camobi de Santa Maria, duas nos dois Centros Comunitários (Santa Helena e Vila Jardim), da mesma área de abrangência da Unidade Básica de Saúde Wilson Noal, e uma palestra na AAPECAN de Santa Maria.

O projeto foi desenvolvido e aplicado pelos acadêmicos do $5^{\circ}$ semestre de graduação do curso de Medicina da Universidade Federal de Santa Maria (UFSM). Para a realização das palestras, foram confeccionados slides no programa Microsoft Power Point ${ }^{\circledR}$ com tópicos sobre a prevenção do câncer, que foram apresentados através de um aparelho de data show. As palestras foram divulgadas, através de cartazes, previamente nos locais em que seriam realizadas. Os dias escolhidos para a realização das palestras foram os dias em que houve as reuniões dos grupos de diabéticos e hipertensos na Unidade e nos centros pertencentes a Wilson Noal. Para o público da AAPECAN, a palestra foi realizada no dia do encerramento da $1^{\text {a }}$ Semana de Prevenção Contra o Câncer, em abril de 2008, evento promovido anualmente pela Associação. Antes das palestras, foram distribuídos questionários aos ouvintes, com questões objetivas relativas ao tema a ser abordado. Junto com o questionário, foram coletados os seguintes dados de cada indivíduo: sexo, idade e escolaridade.

Esse projeto foi aprovado pelo Comitê de Ética em Pesquisa da UFSM (CAAE: 0147.0.243.000-07). Todas as pessoas presentes puderam assistir à palestra, mas apenas os ouvintes que assinaram o termo de consentimento livre e esclarecido responderam ao questionário. Após a palestra, o mesmo questionário foi distribuído aos ouvintes participantes da pesquisa para testar a efetividade das informações dadas. Na análise dos dados, foram excluídos os questionários que não foram corretamente preenchidos.

Os tipos de câncer que foram abordados nas palestras foram escolhidos devido a sua alta incidência no RS (câncer de pulmão, câncer de bexiga, câncer de estômago, câncer de esôfago, câncer de mama, câncer de colo uterino, câncer de cólon e reto, câncer de próstata, câncer de pele, câncer de cavidade oral), segundo dados do INCA (2008). Os temas abordados nas palestras foram: o que é o câncer, tipos de câncer mais incidentes no RS, fatores de risco para cada tipo de câncer (fumo, álcool, sedentarismo, má alimentação, exposição solar sem 
proteção) e como preveni-los (auto-exame das mamas, exame citopatológico, regra ABCD para o autoexame da pele, auto-exame da cavidade oral, atividade física, hábitos alimentares).

Foi realizada dupla digitação das informações coletadas, seguida da análise através de média, frequência e tabelas pelo programa Epi Info versão 6.04.

\section{RESULTADOS E ANÁLISE}

O número de indivíduos participantes dos grupos de diabéticos e hipertensos era de aproximadamente 250 pessoas, mas a presença destes às reuniões mensais foi de apenas 30\%, correspondendo a 75 indivíduos nos três locais abrangidos pela Unidade Básica de Saúde Wilson Noal. O número de pessoas que estiveram presentes no encerramento da $1^{\text {a }}$ Semana de Prevenção Contra o Câncer, na sede da AAPECAN, foi de 40 pessoas. Logo, o número final de indivíduos participantes das palestras foi de 115 pessoas. Trinta e seis pessoas não aceitaram responder aos questionários, totalizando uma amostra de 79 pessoas. Desses, 18 foram excluídos: 10 por não terem preenchido corretamente o questionário; 6 por terem preenchido somente o questionário após as palestras; 2 por não terem preenchido corretamente o termo de consentimento. O perfil educacional dos ouvintes está exposto na tabela 1. As repostas de 61 indivíduos foram analisadas: 25 (41\%) ouvintes na AAPECAN; 13 (21\%) ouvintes no Centro Comunitário Vila Jardim; 16 (26\%) ouvintes na Unidade de Saúde Wilson Noal e 7 (11,5\%) ouvintes no Centro Comunitário Santa Helena.

Tabela 1: Perfil dos ouvintes das palestras nos quatro locais.

\begin{tabular}{c|c|c}
\hline Variável & Número de ouvintes & Percentual de ouvintes (\%) \\
\hline Sexo feminino & 52 & 85,24 \\
Sexo masculino & 9 & 14,75 \\
Ensino Fundamental incompleto & 22 & 36,06 \\
Ensino Fundamental completo & 8 & 13,11 \\
Ensino médio completo & 26 & 42,62 \\
Ensino superior completo & 3 & 4,91 \\
Idade inferior a 40 anos & 29 & 40,98 \\
Idade superior a 40 anos & 32 & 59,01 \\
\hline Total de ouvintes & 61 & \\
\hline
\end{tabular}

Fonte: elaborada pelas autoras, 2009. 
Conforme observamos na tabela 2, nas questões sobre o câncer de bexiga, de colo de útero, de cavidade oral, de estômago, de esôfago, de mama e de próstata houve um maior número de acertos após a palestra. Não foi calculada a significância, pois havia caselas com $n<5$. Nas questões sobre câncer de pele e intestino, houve um leve decréscimo no número de acertos após a palestra. Na questão sobre câncer de pulmão não houve melhora nem piora após a palestra.

Tabela 2: Desempenho dos participantes nos questionários.

\begin{tabular}{c|c|c|c}
\hline $\begin{array}{c}\text { Tipo de câncer } \\
\text { abordado na questão }\end{array}$ & $\begin{array}{c}\mathrm{N}^{\circ} \text { de pessoas que } \\
\text { acertaram a questão } \\
\text { no pré-teste }\end{array}$ & $\begin{array}{c}\mathrm{N}^{\circ} \text { de pessoas que } \\
\text { acertaram a questão } \\
\text { no pós-teste }\end{array}$ & $\begin{array}{c}\text { Aumento percentual } \\
\text { no número de acertos } \\
\text { após a palestra }\end{array}$ \\
\hline Bexiga & 19 & 45 & $42,62 \%$ \\
Boca & 56 & 59 & $4,91 \%$ \\
Colo Uterino & 48 & 51 & $4,91 \%$ \\
Esôfago & 57 & 59 & $3,27 \%$ \\
Estômago & 52 & 58 & $9,83 \%$ \\
Intestino & 53 & 52 & $-1,63 \%$ \\
Mama & 30 & 56 & $42,62 \%$ \\
Pele & 58 & 56 & $-3,27 \%$ \\
Próstata & 55 & 55 & $27,86 \%$ \\
Pulmão & 55 & $0 \%$ \\
\hline
\end{tabular}

Fonte: elaborada pelas autoras, 2009.

\section{CONSIDERAÇÕES FINAIS}

A experiência discente foi relevante, destacando-se o contato dos alunos da graduação com as pessoas leigas sadias e doentes, assim como a percepção pelos alunos da necessidade de adequação da linguagem de informação e a sua compreensão da realidade de desconhecimento acerca da prevenção do câncer pelo público participante. Notamos um maior desconhecimento inicial sobre a prevenção dos cânceres de mama $(n=30)$ e próstata $(n=5)$, o que justifica a maior divulgação de campanhas nacionais de informação e 
rastreamento da população (INCA, 2008). A região sul apresenta uma maior incidência relativa de câncer de intestino grosso (INCA, 2008), devido ao hábito do consumo elevado de carnes. Nesse estudo, não foi verificado um índice baixo de número de ouvintes que responderam corretamente a questão sobre o tema, tanto antes (n=53) quanto após (n=52) a palestra, indicando que o grau de informação deste público é mais adequado. Observamos o mesmo dado em relação à pergunta referente ao câncer de pulmão. O tabagismo é um conhecido fator de risco para o câncer de pulmão neste público, sendo entendida a importância do combate a este vício para a prevenção deste câncer. Conforme Doll (2004, p.1.519), o câncer de pulmão e a doença pulmonar obstrutiva crônica estão intimamente relacionados com o hábito contínuo de fumar e com o número diário de cigarros consumidos. Além disso, os fumantes perdem cerca de 10 anos de vida em relação aos não fumantes. Há uma proteção substancial até mesmo para os fumantes que abandonam o hábito somente na $5^{\text {a }}$

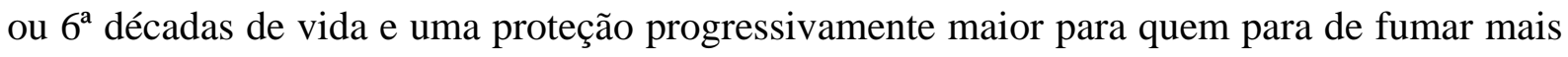
cedo. Também não observamos diferença em relação ao câncer de pele, mas medidas de proteção aos raios ultravioleta foram colocadas, assim como a diferenciação clínica de tumores do tipo melanoma de não melanoma. Apesar da ampla divulgação da importância do rastreamento do câncer de colo uterino, notamos que a informação referente ao exame Papanicolau e sua frequência foram extremamente úteis (INCA, 2008).

Um projeto semelhante de intervenção primária nos serviços de atenção básica foi realizado entre os anos de 1997 e 1999, no estado do Maranhão (Brasil). Foram realizadas palestras à população sobre prevenção e diagnóstico precoce do câncer oral. Cada um dos participantes foi avaliado antes e depois das palestras através de um questionário. Como resultado, houve um avanço significativo de conhecimentos sobre a importância da prevenção e do diagnóstico precoce dessa patologia, evidenciando que é imprescindível a realização de grandes campanhas de esclarecimento junto à população, ampliando, dessa forma, a curabilidade e o controle do câncer (THOMAZ, 2000). No nosso projeto, foi notada uma necessidade de maior esclarecimento sobre as causas e a apresentação clínica do câncer de cavidade oral. Foi enfatizado o esclarecimento sobre a etiologia ligada ao tabagismo e etilismo, assim como a apresentação de lesões indolores que podem ser detectadas pelo paciente ou pelo seu dentista (DUNCAN, 2004).

As palestras de prevenção se mostraram muito úteis à população leiga destes grupos de Santa Maria, no entanto precisamos adaptar continuamente a forma de exposição conforme o nível educacional dos expectadores, que neste caso foi significativamente baixo, sendo que 
36\% dos participantes apresentavam ensino fundamental incompleto. Visando esse público de menor escolaridade, o ideal é que as palestras sejam ministradas de maneira informal, utilizando os slides apenas como ilustração, com muito pouco texto. Assim, o público geral se sente mais à vontade para participar com perguntas e para realizar outras intervenções. Pelo desconhecimento inicial do público abordado, no nosso projeto, adaptamos o material segundo essa necessidade após a realização da primeira palestra.

Para Holland e Frei (2003), a melhor maneira de reduzir mortes por câncer é através da detecção precoce. Adotar estilos de vida saudáveis é importante. A prevenção ao câncer é também uma ótima maneira de reduzir custos. Os tratamentos curativos estão bem acima do orçamento de muitos países (STEWART; COATES, 2005). Com o envelhecimento crescente da população, a redução das taxas de mortalidade, a detecção precoce das doenças e os progressos no tratamento de várias enfermidades, é fundamental enfatizar a prevenção primária. Caso contrário, a prevalência e a morbidade associada às principais doenças crônicas aumentarão e consumirão uma parcela ainda maior dos recursos médicos disponíveis (GOLDMAN; AUSIELLO, 2005).

\section{AGRADECIMENTOS}

Agradecemos a importante colaboração de Marinel Mór Dall’Agnol, médica epidemiologista e professora assistente do Departamento de Saúde da Comunidade do Centro de Ciências da Saúde da UFSM, por ter colaborado na execução e na análise dos dados deste trabalho. Também agradecemos aos acadêmicos de Medicina da Universidade Federal de Santa Maria, Francine Teixeira, Lisete Fronza, Lisiane Vargas e Elemar da Silva Resch que participaram deste projeto de extensão e ajudaram significativamente na execução do mesmo.

\section{REFERÊNCIAS}

CURRY, S. et al. Fulfilling the Potential of Cancer Prevention and Early Detection. Washington, DC: National Academy Press, 2003. In: STEIN, C. J.; COLDITZ, G. A. Modifiable risk factors for cancer. British Journal of Cancer, UK, v. 90, p. 299-303, jan. 2004. Disponível em: <http://www.nature.com/bjc/index.html>. Acesso em: 04 jun. 2008.

DOLL, R. et al. Mortality in relation to smoking: 50 years' observations on male British doctors. British Medical Journal, UK, v.328, n.7455, p.1519, jun. 2004. Disponível em: $<$ http://www.bmj.com/>. Acesso em: 25 jun. 2009. 
DUNCAN, B. et al. Medicina Ambulatorial: Condutas de Atenção Primária Baseadas em Evidências. 3. ed. Porto Alegre: Artmed, 2004. p. 496, 497, 839, 840.

FERNANDES, C.E., BARACAT, E. C., LIMA, G. R. Climatério: manual de orientação. São Paulo: Ponto, 2004. p.179.

GOLDMAN L.; AUSIELLO, D. Cecil. Tratado de Medicina Interna. 22. ed. Rio de Janeiro: Elsevier, 2005. v. 1. p. 49, 55.

HOLLAND; FREI. Cancer Medicine. 6. ed. Hamiltom: BC Decker Inc, 2003. v. 1. p. 397, 423.

INSTITUTO BRASILEIRO DE CÂNCER (INCA). Estimativa 2008: Incidência de câncer no Brasil. Disponível em: <http://www.inca.gov.br/>. Acesso em: 19 set. 2008.

Programa Nacional de Controle do Câncer do Colo do Útero e de Mama - Viva Mulher. Disponível em: <http://www.inca.gov.br/>. Acesso em: 19 set. 2008.

. Detecção do Câncer de Próstata. Disponível em: <http://www.inca.gov.br/>. Acesso em: 19 set. 2008.

ORGANIZAÇÃO MUNDIAL DE SAÚDE (OMS). World Cancer Report 2008. Disponível em: <http://www.who.int/en/>. Acesso em: 14 maio 2009.

. Global Action Against Cancer - Updated Edition, 2005. Disponível em:

$<$ http://www.who.int/en/>. Acesso em: 14 maio 2009.

. Causes of cancer in the world: comparative risk assessment of nine behavioural and environmental risk factors. Disponível em: <http://www.who.int/en/>. Acesso em: 14 maio 2009.

STEWART, B. W.; COATES, A.S. Cancer prevention: A Global Perspective. Journal of Clinical Oncology, New York, v. 23, n. 2, p. 392-403, jan. 2005.

THOMAZ, E. et al. A importância da educação como estratégia para prevenção e diagnóstico precoce do câncer oral. Acta Oncológica Brasileira, São Paulo, v. 20, n. 4, p. 149-152, out.dez. 2000. 